Pacific Journal of Mathematics

BOREL SELECTORS FOR SEPARATED QUOTIENTS 


\title{
BOREL SELECTORS FOR SEPARATED QUOTIENTS
}

\author{
Douglas E. Miller
}

\begin{abstract}
We use a nonstandard version of the Kuratowski, RyllNardzewski general selection theorem to establish the existence of Borel measurable selectors for certain equivalence relations on Polish spaces.
\end{abstract}

The two main results of this paper establish the existence of Borel measurable selectors of determined complexity for certain equivalence relations on Polish spaces.

THEOREM A. Let $\boldsymbol{G}$ be a Polish topological group which acts continuously on a Polish space $\boldsymbol{Y}$ inducing an equivalence $\boldsymbol{E}$ on $\boldsymbol{Y}$. Suppose $\boldsymbol{A} \subseteq \boldsymbol{Y}$ is an invariant Borel set of ambiguous class $\alpha \geqq 0$ such that quotient Borel space $\boldsymbol{A} / \boldsymbol{E}$ is countably separated by projections of invariant sets of ambiguous class $\alpha$. Then there is an $\alpha$-Borel measurable selector function for $E$ on $A$.

Given $\gamma \in \omega_{1}$, let $\gamma^{*}=\sup (\gamma+\beta: \beta<\gamma)$, so $\gamma^{*}=\gamma+\beta$ when $\gamma=\beta+1, \gamma^{*}=\gamma \cdot 2$ when $\gamma$ is a limit ordinal.

Theorem B. Let $\boldsymbol{Y}$ be a Polish space and let $E$ be an equivalence on $Y$ whose equivalence classes are $G_{o}$ sets. Suppose that the $E$ saturation of each open set in a given basis is of ambiguous class $\gamma>0$. Then there is a $\gamma^{*}$-Borel selector function for $E$ on $\boldsymbol{Y}$.

This work was stimulated by a recent paper of Burgess [1] in which some techniques from Vaught [11] were used to obtain a Borel selector in the case $\boldsymbol{A}=\boldsymbol{Y}$ of Theorem A. The selector in [1] is obtained via an application of Suslin's theorem, so its complexity is very hard to estimate. We will use Vaught's method in a different way to provide a more direct (and simpler) construction in which the complexity of the selector is apparent.

Burgess's result extends a theorem of E. Effros [2] on locally compact groups. Theorem A will be proved as a special case of a more general result (3.2) about Borel actions. The existence of a Borel selector for noncontinuous actions appears to be new in all cases.

The existence of a Borel selector (of unknown complexity) under the hypothesis of Theorem B is a recent result of S. M. Srivastava [10]. The special case, $\gamma=1$, was previously established by the present author in [8]. These results extend earlier work by Kallman and Mauldin [3] and Kuratowski and Maitra [5]. Theorem B is a 
special case of a slightly stronger result (3.4) with a weaker requirement on the equivalence classes.

Our arguments are based on a "nonstandard" version of the well-known general theorem on selectors of Kuratowski and Ryll-Nardzewski. This result is proved in $\S 1$. Section 2 collects some material concerning topologies generated by Borel sets and the main results are proved in $\S 3$.

Throughout the paper we reserve boldface notation ( $\boldsymbol{Y}$, etc.) for topological spaces. Formally, we regard $Y$ as a pair $(Y, \mathscr{T})$ where $Y$ is the underlying set and $\mathscr{T}$ is the collection of open subsets of $\boldsymbol{Y}$. However, we make free use of the common identification of $\boldsymbol{Y}$ with $Y$ when no confusion will result (as in the previous sentence). $\boldsymbol{X} \times \boldsymbol{Y}$ is $X \times Y$ with the product topology; $\boldsymbol{A} \subseteq \boldsymbol{Y}$ is $A$ with the relative topology; $2^{\omega}$ is $2^{\omega}$ with the product topology (i.e., the Cantor space).

The author wishes to thant Professor R. L. Vaught for many helpful comments on a preliminary version of this paper.

1. Measurable choice functions. Suppose $Y=(Y, \mathscr{T})$ is a topological space, $X$ is an arbitrary set, and $F$ is a function on $X$ to the power set $\mathscr{P}(\boldsymbol{Y})$. A choice function for $F$ is a map $f: X \rightarrow Y$ such that $f(x) \in F(x)$ for each $x \in X$. (We reserve the common term "selector" for its other meaning connected with equivalence relations.) Given $\mathscr{D} \subseteq \mathscr{P}(X)$, we say that $f$ is $\mathscr{D}$-measurable provided $f^{-1}(O) \in$ $\mathscr{D}$ whenever $O \in \mathscr{T} . \mathscr{D}_{\sigma}$ is the countably additive family generated by $\mathscr{D}$.

An operator $\#: \mathscr{T} \rightarrow \mathscr{P}(X)$ is countably additive provided $\bigcup_{i \in \omega}\left(O_{i}^{\sharp}\right)=\left(\bigcup_{i \in \omega} O_{i}\right)^{\#}$ whenever $\left\{O_{i}: i \in \omega\right\} \subseteq \mathscr{T}$. \# is a semisaturation operator for $F$ provided

(i) $Y^{\sharp}=X$

(ii) $O^{\#} \subseteq\{x$ : $F(x) \cap O \neq \varnothing\}$ for each $O \in \mathscr{I}$.

The simplest example of an additive semisaturation operator is obtained by setting $O \#=O^{+}=\{x: F(x) \cap O \neq \varnothing\}$. Two further examples will play essential roles in $\S 3$. The standard case “\# $=+$ " of Theorem 1.1 is the main result of Kuratowski and Ryll-Nardzweski [6]. Our proof is abstracted from theirs.

THeOREm 1.1. Assume that $Y$ is a Polish space, $F$ is function with domain $X$ whose values are closed subsets of $Y$ and $\#: \mathscr{T} \rightarrow \mathscr{P}(X)$ is a countably additive, semisaturation operator for $F$. Suppose that $\mathscr{D}$ is a field of subsets of $X$ such that for every $O \in \mathscr{T}, O^{*} \in \mathscr{D}_{\text {o }}$. Then there is a $\mathscr{D}_{\sigma}$-measurable choice function for $F$.

Proof. For $U \subseteq Y$, let $\bar{U}$ be its closure. Fix a complete metric 
$d$ for $\boldsymbol{Y}$ which is bounded by 1 , and let $\mathscr{H}$ be a countable basis for $Y$ with $Y \in \mathscr{K}$. We also fix an enumeration of $\omega \times \omega$ as $\left\{\left(a_{i}, b_{i}\right): i \in \omega\right\}$.

We enumerate $\mathscr{C}$ as $\left\{U_{n}: n \in \omega\right\}$ such that $U_{0}=Y$ and any singletons are listed infinitely often. For each $n$, we choose a sequence $\left\{B_{n, k}: l i \in \omega\right\} \subseteq \mathscr{D}$ such that $U_{n}^{\sharp}=\bigcup_{k} B_{n, k}$. We also define

$$
I_{n}=\left\{m>n \text { : diameter }\left(\bar{U}_{m}\right)<1 / 2 n \text { and } \bar{U}_{m} \leqq U_{n}\right\} \text {. }
$$

It follows that $U_{n}=\bigcup_{m \in I_{n}} U_{m}$.

For each $x \in X$, we define, by induction on $n$, a sequence $\left\{p_{n}(x): n \in \omega\right\}$ satisfying:

(a) $p_{n}(x) \geqq n$ and $p_{n+1}(x) \in I_{p_{n}}(x)$

(b) $x \in U_{p_{n}(x)}^{\sharp}$.

The construction is started by setting $p_{0}(x)=0$. Now assume that $p_{n}(x)=p_{n}$ is given; we show how to define $p_{n+1}(x)$.

By condition (b) and the fact that $\#$ is countably additive, $x \in$ $\bigcup_{m \in I_{p_{n}}} U_{m}^{\sharp}=\mathbf{U}_{m \in I_{p_{n}}} \bigcup_{k \in \omega} B_{m, k}$. Let $i$ be the first natural number such that $a_{i} \in I_{p_{n}}$ and $x \in B_{a_{i}, b_{i}}$. We set $p_{n+1}(x)=a_{i}$. Clearly (b) holds and since $a_{i} \in I_{p_{n}}, a_{i}>p_{n} \geqq n$ so (a) holds as well.

Now fix $x$ and write $p_{k}=p_{k}(x)$ for each $k \in \omega . \quad B y$ (a) and the definition of the sets $I_{n}$, we have $U_{p_{0}} \supseteqq \bar{U}_{p_{1}} \supseteqq U_{p_{1}} \supseteqq \bar{U}_{p_{2}} \supseteqq \cdots$ with each $\operatorname{diam}\left(\bar{U}_{p_{k}}\right)<1 / k$. It follows that $\bigcap_{k \in \omega} U_{p_{k}}$ is a singleton. We define $f(x)$ to be the unique element of $\bigcap_{k} U_{p_{k}}$. We must show that $f$ has the required properties.

By (b), for each $k, x \in U_{p_{k}}^{\sharp} \subseteq U_{p_{k}}^{+}$. Thus, each $F(x) \cap U_{p_{k}} \neq \varnothing$. It follows that $f(x)$ is the limit of a convergent sequence from $F(x)$. Since $F(x)$ is closed, $f(x) \in F(x)$. Thus $f$ is a choice function for $F$.

It remains to show that $f$ is $\mathscr{D}_{\sigma}$-measurable. First we prove a lemma:

$$
\text { For each } n, f^{-1}\left(U_{n}\right)=\bigcup_{m \in I_{n}} \bigcup_{k \leqq m}\left\{x: m=p_{k}(x)\right\} .
$$

Inclusion from right to left is obvious since $m=p_{k}(x)$ implies $f(x) \in U_{m} \subseteq U_{n}$. For the reverse inclusion suppose $f(x) \in U_{n}$. Since $U_{n}$ is open we may choose $k>n$ such that $\{y: d(f(x), y)<1 / k\} \subseteq U_{n}$. Since $\operatorname{diam}\left(U_{p_{k}}(x)\right)<1 / k$ and $f(x) \in U_{p_{k}(x)}$, we have $U_{p_{k}(x)} \leqq U_{n}$. Thus $p_{k}(x) \in I_{n}$ and the lemma is established.

Now set $C_{k, m}=\left\{x: m=p_{k}(x)\right\}$. By (1) it suffices to show that each $C_{k, m}$ belongs to $\mathscr{D}_{\sigma}$. This is easily checked by induction on $k$ using the assumption that $\mathscr{D}$ is a field of sets, together with the observation:

$$
p_{k+1}(x)=m \text { if and only if }
$$

(2) $\quad(\exists r<m)\left[m \in I_{r}\right.$ and $p_{k}(x)=r \quad$ and $\quad(\exists i)\left(m=a_{i} \quad\right.$ and $x \in B_{a_{i}, b_{i}}$ and $\quad(\forall j<i)\left(a_{j} \notin I_{r}\right.$ or $\left.\left.x \notin B_{a_{j}, b_{j}}\right)\right]$. 
REMARK. Note that if we drop the assumption that each $F(x)$ is closed, the construction still provides a map $f$ with the property that $f(x) \in F(x)$ whenever $F(x)$ closed.

2. Borel-generated topologies. The second set of observations that we require deals with the notion of Borel generated topologies on Polish spaces. It is well-known that if B is a Borel subset of a Polish space $\boldsymbol{Y}$, then there is a topology on $Y$ compatible with the Borel structure on $Y$ in which $B$ is both closed and open (cf. [4], p. 448). In this section we examine this remark with special concern for the Borel complexity of the sets generating the new topology on $Y$. Our approach is closely akin to the techniques from mathematical logic of closing a set of formulas under subformulas and of adding Skolem predicates to a language.

Throughout this section, $\boldsymbol{Y}=(Y, \mathscr{T})$ is a fixed Polish space. $\mathscr{H}$ is a fixed countable basis for $Y$ and $\mathscr{B}$ is the collection of Borel subsets of $Y . \quad S_{\alpha}, M_{\alpha}$, and $A_{\alpha}$ respectively denote the $\alpha$ th additive, multiplicative and ambiguous classes in the Borel hierarchy on $Y$ (so $S_{0}=\mathscr{T}, S_{1}=F_{\sigma}, A_{1}=F_{o} \cap G_{o}$, etc.). A function $s: Y \rightarrow Z$ is a $\alpha$-Borel provided $S^{-1}(0) \in S_{\alpha}$ whenever 0 is open in $Z$.

For any Borel set $B$ define $r(B)$ to be the least ordinal $\alpha$ such that $B \in A_{\alpha}$. We say that a collection $\mathscr{C}$ of Borel sets is supported provided $\mathscr{C}$ is closed under complementation and for all $B \in \mathscr{C}$, if $r(B)=\alpha>1$ then there exist collections $\left\{B_{i j}: i, j \in \omega\right\}$ and $\left\{C_{i j}: i, j \in \omega\right\}$ included in $\mathscr{C} \cap \mathrm{U}_{\beta<\alpha} A_{\beta}$ such that

$$
B=\bigcap_{i} \bigcup_{j} B_{i j}=\bigcup_{i} \bigcap_{j} C_{i j} .
$$

Lemma 2.1. Suppose $\alpha \geqq 1$. Let $\mathscr{S}$ be a countable collection of $A_{\alpha}$ sets which is closed under complementation. Then there exists a countable collection $\mathscr{C} \subseteq \bigcup_{\beta<\alpha} A_{\beta}$ such that $\mathscr{C} \cup \mathscr{S}$ is supported.

Proof. We proceed by induction on $\alpha$. The case $\alpha=1$ is treated trivially by setting $\mathscr{C}=\varnothing$.

Let $\alpha$ and $\mathscr{S}$ be given with $\alpha>1$ and $\mathscr{S}=\left\{R_{n}: n \in \omega\right\}$. Assume that the lemma holds for all $\beta<\alpha$. For each $n \in \omega$ choose sets $B_{i j}^{n}$, $C_{i j}^{n} \in \bigcup_{\beta<\alpha} A_{\beta}(i, j \in \omega)$ such that

$$
R_{n}=\bigcap_{i} \bigcup_{j} B_{i j}^{n}=\bigcup_{i} \bigcap_{j} C_{i j}^{n} .
$$

Applying the induction hypothesis, choose for each triple $n, i, j$ a supported collection $\mathscr{C}_{i j}^{n} \subseteq \bigcup_{B<\alpha} A_{\beta}$ such that $B_{i j}^{n}, C_{i j}^{n} \in \mathscr{C}_{i j}^{n}$. It is evident that $\mathscr{C}=\mathbf{U}_{n} \mathbf{U}_{i} \mathbf{U}_{j} \mathscr{C}_{i j}^{n}$ has the required property.

LEMma 2.2. Suppose $\mathscr{S} \subseteq \mathscr{B}$ is countable and supported. Let 
$\mathscr{J}^{\prime}$ be be the topology generated by $\mathscr{S} \cup \mathscr{T}$. Then $\boldsymbol{Y}^{\prime}=\left(Y, \mathscr{T}^{\prime}\right)$ is Polish.

Proof. Enumerate $\mathscr{S}$ as $\left\{B_{\imath}: i \in \omega\right\}$. Given $y \in Y$ define $\xi_{y} \in 2^{\omega}$ by the equation $\xi_{y}(i)=1 \Leftrightarrow y \in B_{i}$. Define $\boldsymbol{G}=\left\{\left(y, \xi_{y}\right): y \in Y\right\} \subseteq$ $Y \times 2^{\prime \prime}$. The map $f: Y^{\prime} \rightarrow G$ defined by $f(y)=\left(y, \xi_{y}\right)$ is apparently one-to-one and onto. Since for each $i$,

$$
f\left(B_{i}\right)=G \cap\{(y, \xi): \xi(i)=1\}
$$

and for each $O \in \mathscr{G}, f(O)=G \cap\{(y, \xi): y \in O\}, f$ is open. Since $f^{-1}(O \times\{(y, \xi): \xi(i)=k\})$ is either $O \cap B_{i}$ or $O \cap\left(\sim B_{i}\right)($ as $k=0$ or 1$)$ and $\mathscr{S}$ is closed under complementation, $f$ is continuous. Thus, $Y^{\prime}$ is homeomorphic to $G$. Since any $G_{\hat{o}}$ subspace of a Polish space is Polish, it suffices to show that $G$ is $G_{o}$ in $Y \times 2^{\prime \prime}$.

For each $i \in \omega$ we define a $G_{j}$ set $G_{i}$ as follows: If $r\left(B_{i}\right)>1$ we use the fact that $\mathscr{P}$ is supported to choose functions $p_{i}, q_{i}$ such that

$$
B_{i}=\bigcap_{i} \bigcup_{1} B_{p_{i}(l, 1)}=\sim \bigcap_{i} \bigcup_{1} B_{q_{i}(k, 1)}
$$

with each $r\left(B_{p_{i}(k, 1)}\right), r\left(B_{q_{\imath}(k, 1)}\right)<r\left(B_{\imath}\right)$. In this case we define

$$
\begin{aligned}
G_{i}= & \left\{(y, \xi): \xi(i)=1 \text { and }(\forall k)(\exists l)\left(\xi\left(p_{i}(k, l)=l\right)\right)\right\} \\
& \cup\left\{(y, \xi): \xi(i)=0 \text { and }(\forall k)(\exists l)\left(\xi\left(q_{i}(k, l)=l\right)\right)\right\} .
\end{aligned}
$$

If $r\left(B_{i}\right) \leqq 1$ we choose open sets $U_{k}^{i}, V_{l}^{i}$ such that

then define

$$
B_{i}=\bigcap_{i} U_{k}=\sim \bigcap_{b} V_{i}
$$

$$
\begin{aligned}
G_{\imath}= & \left\{(y, \xi): \xi(i)=1 \text { and }(\forall k) y \in U_{k}\right\} \\
& \cup\left\{(y, \xi): \xi(i)=0 \text { and }(\forall k) y \in V_{k}\right\} .
\end{aligned}
$$

A straightforward induction on $r\left(B_{i}\right)$ shows

(a) For all $y \in Y,\left(y, \xi_{y}\right) \in G_{i}$ and

(b) For all $(y, \xi) \in \cap G_{i}, \xi(i)=\xi_{y}(i)$.

Thus $G=\bigcap_{\imath} G_{i}$ and the lemma is proved.

Combining 2.1 and 2.2 we obtain

Theorem 2.3. Suppose $\mathscr{Y} \subseteq A_{\alpha}$ is countable and closed under complementation with $\alpha \geqq 1$. Then there is a countable collection $\mathscr{C} \subseteq \bigcup_{\beta<\alpha} A_{\beta}$, such that $\mathscr{H} \cup \mathscr{S} \cup \mathscr{B}$ generates a Polish topology on $Y$.

3. Selectors. Suppose $E$ is an equivalence relation on a space $A$. We write $A / E$ for the set of $E$-equivalence classes and 
denote the associated projection map $\pi: y \mapsto[y]$. A selector for $\boldsymbol{E}$ on $\boldsymbol{A}$ is a map $\boldsymbol{s}: \boldsymbol{A} \rightarrow \boldsymbol{A}$ satisfying (i) $(\forall y)(s(y) \in[y])$ and (ii) $\left(\forall y_{1} y_{2}\right)\left(y_{2} \in\left[y_{1}\right] \Rightarrow s\left(y_{1}\right)=s\left(y_{2}\right)\right)$. A transversal for $E$ is a set which intersects each equivalence class in a singleton. Note that if $s$ is a selector for $E$, then the fixed points of $s$ form a transversal $T_{s}$ for $E$. If $s$ is $\alpha$-Borel, then $T_{s}$ is an $M_{\alpha}$ subset of $A$. Given $B \subseteq A$, we say that a collection $\mathscr{S}$ of $E$-invariant sets separates $B / E$ provided $(\forall y \in B)([y]=\bigcap\{S \in \mathscr{S}:[y] \subseteq S\})$.

As promised, we will use the constructions of $\S 1$ and $\S 2$ to obtain two results on the existence of Borel selectors for certain equivalence relations on Polish spaces. In each case we introduce a Borel-generated topology on the relevant space $\boldsymbol{A}$ in which the equivalence classes are closed. We then consider the identity map $F: A / E \rightarrow \mathscr{P}(\boldsymbol{A})$ and introduce a semisaturation operator derived from the constructions of Vaught [11]. Theorem 1.1 provides a choice function $f$ for $I, f \circ \pi$ is the required selector.

A. Borel actions. Throughout this subsection, $G$ is a nonmeager topological group with a countable basis. In the most notable case, $\boldsymbol{G}$ is Polish. $\boldsymbol{Y}$ is a Polish space and $J: G \times \boldsymbol{Y} \rightarrow \boldsymbol{Y}$ is a Borel measurable function. For $g \in G, J^{g}$ is the function $y \mapsto J(g, y)=g y$. We assume that $J$ defines an action of $G$ on $Y$, i.e., that the map $g \mapsto J^{g}$ is a homomorphism from $G$ to the group of permutations of $\boldsymbol{Y}$. The action induces the equivalence relation $\left.E_{J}=\{y, g y): y \in \boldsymbol{Y}, g \in \boldsymbol{G}\right\}$ on $Y$.

Following Vaught [11] we write, for $B \subseteq Y, y \in Y B^{y}=\{g: g y \in B\}$, $B^{+}=\left\{y: B^{y} \neq \varnothing\right\}, B^{4}=\left\{y: B^{y}\right.$ is meager $\}$.

It follows from the definitions and the fact that $G$ is a Baire space that for $A, B, B_{i} \subseteq Y$.

(2) $B^{\Delta}$ is $E_{J}$-invariant and $B^{\Delta} \subseteq B^{+}$.

(3) $\left(\bigcup_{i \in \omega} B_{i}\right)^{4}=\bigcup_{i \in \omega} B_{i}^{\Delta}$.

(4) If $A$ is invariant then $A^{4}=A$ and $(A \cap B)^{4}=A \cap B^{4}$.

The following lemma is proved in [11] for the case $\gamma=0$ and in [7] for the general case. (In [7] it is asserted only that $B^{1}$ is Borel, but the argument establishes the stronger result 3.1.)

Lemma 3.1. Suppose $J$ is $\gamma$-Borel and $B \in S_{\beta}$. Then $B^{\Delta} \in S_{\gamma+\beta}$.

THEOREM 3.2. Let $\alpha \geqq 1, \gamma \geqq 0$. Assume that $J$ is a $\gamma$-Borel action on $Y$. Suppose $A \subseteq Y$ is an invariant $A_{\alpha}$ set and that $\mathscr{S}$ is a countable collection of invariant $A_{\alpha}$ sets which separates $A / E_{J}$. Then there exists a $\gamma+\alpha$-Borel selector for $E$ on $\boldsymbol{A}$.

Proof. We assume without loss of generality that $A \in \mathscr{S}$ and 
that $\mathscr{S}$ is closed under complementation and finite intersection. By 2.3 we can choose a countable set $\sigma^{\prime} \subseteq \bigcup_{\beta<\alpha} A_{\beta}$ such that $\mathcal{Y} \cup \sigma^{\prime} U$ $\mathscr{\mathscr { H }}$ generates a Polish topology $Y^{-\prime}$ on $Y$. Write $Y^{\prime}=\left(Y, Y^{\prime}\right)$. Let $\mathfrak{B}$ be the closure of $\mathscr{C} \cup \mathscr{H}$ under finite intersections. Then $Y^{\prime}$ has the basis : $\mathscr{H}^{\prime \prime}=\{B \cap S: B \in \mathfrak{B}, S \in \mathscr{S}\}$.

Let $A^{\prime}$ be $A$ with the relative topology from $I^{\prime} \cdot A^{\prime}$ has the basis $\mathscr{\varkappa}=\left\{K \in \mathscr{Y}^{\prime}: K \subseteq A\right\}$. Since $A$ is closed in $\mathscr{Y}^{\prime}, \boldsymbol{A}^{\prime}$ is Polish.

Set $X=A / E$ and let $F$ be the the identity function from $X$ to $\mathscr{S}\left(\boldsymbol{A}^{\prime}\right)$. For $O \subseteq \boldsymbol{A}^{\prime}$ define $O^{*}=\pi\left(O^{4}\right)$. It follows from (2) and (4) that $\#$ is a semisaturation operator for $F$ and from (3) that $\#$ is countably additive.

Let $\mathscr{D}$ be the field generated by $\left\{K^{\prime}: K \in \mathscr{K}^{\prime}\right\}$ and let $\mathscr{D}^{\prime}=$ $\left\{\pi^{-1}(D): D \in \mathscr{D}\right\}$. Since each $K^{4}$ is invariant, $\mathscr{D}^{\prime}$ is just the field generated by $\left\{K^{\Delta}: K \in \mathscr{K}\right\}$. Each $K \in \mathscr{K}$ has the form $B \cap S$ with $S$-invariant $A_{\alpha}$ and $B \in \bigcup_{\beta<\alpha} S_{\beta}$; by (4) and 3.1, $K=B \cap S \in A_{\tau+\alpha}$.

It follows that

$$
\mathscr{D}^{\prime} \subseteq A_{i+\alpha} .
$$

Since $\mathscr{S}$ separates $A / E_{J}$, each $[y]$ is closed in $A^{\prime}$. Thus, we may apply 1.1 to obtain a $\mathscr{D}_{\sigma}$-measurable choice function $f: A / E_{J} \rightarrow \boldsymbol{A}^{\prime}$. Define $s=f \circ \pi$. Clearly, $s$ is a selector for $E$ on $A$. We claim that $s$ is $\gamma+\alpha$-Borel with respect to $\boldsymbol{A}$. Since $\boldsymbol{A}^{\prime}$ refines $\boldsymbol{A}$ it suffices to establish: $s$ is a $\gamma+\alpha$-Borel map from $\boldsymbol{A}$ to $\boldsymbol{A}^{\prime}$.

Consider an arbitrary open set $O \subseteq \boldsymbol{A}^{\prime}$. Since $f$ is $\mathscr{D}_{\sigma}$-measurable we may choose $D_{i} \in \mathscr{D}$ such that $f^{-1}(O)=\bigcup_{i \in \omega} D_{i}$. Then $s^{-1}(O)=$ $\pi^{-1}\left(f^{-1}(O)\right)=\bigcup_{\imath \in \omega} \pi^{-1}\left(D_{\imath}\right)$. By $(5)$ each $\pi^{-1}\left(D_{i}\right) \in A_{\gamma+\alpha}$ so $s^{-1}(O) \in S_{\gamma+\alpha}$ as required.

B. Equivalence relations with relatively large equivalence classes. Assume throughout this subsection that $E$ is an equivalence relation on a Polish space $Y$, such that each equivalence class is Borel in $Y$ and a Baire space in its relative topology. In the most notable special case, each equivalence is $G_{0}$ in $Y$. For $B \subseteq Y$ we continue to write $B^{+}=\{y:[y] \cap B \neq \varnothing\}$.

$\mathscr{H}$ is a fixed countable basis for $Y$ with $Y \in \mathscr{C} . U, V$ will always denote elements of $\mathscr{K}$. We write

$$
\mathscr{S}=\left\{U^{+}: U \in \mathscr{C}\right\} \cup\left\{\sim U^{+}: U \in \mathscr{K}\right\} .
$$

Lemma 3.3 (Compare [8] Lemma 2.) $\mathscr{S}$ separates $Y / E$.

Proof. Given $y \in Y$ let 


$$
\mathscr{S}_{y}=\left\{U^{+}: U \cap[y] \neq \varnothing\right\} \cup\left\{\sim\left(U^{+}\right): U \cap[y]=\varnothing\right\} .
$$

We must show $[y]=\cap \mathscr{S}_{y}$.

Inclusion from left to right is immediate from the definition of $\mathscr{S}_{y}$. For the reverse inclusion suppose $x \notin[y]$.

There are two cases to consider.

Case 1. $[x] \nsubseteq \overline{[y]}$ (the closure of $[y]$ ).

Let $z \in[x], z \notin[y]$. Then for some $U \in \mathscr{H}, z \in U$ and $U \cap[y]=\dot{\phi}$. Then $\sim\left(U^{+}\right) \in \mathscr{S}_{y}$ and $x \notin \sim\left(U^{+}\right)$so $x \notin \cap \mathscr{S}_{y}$.

Case 2. $[x] \subseteq[\overline{[}]$.

We claim that $[x]$ is not dense in $[y]$. This suffices since we then obtain $U \in \mathscr{H}$ with $U \cap[y] \neq \varnothing, U \cap[x]=\varnothing$ so $U^{+} \in \mathscr{S}_{y}$ and $x \notin U^{+}$. To verify the claim suppose arguendo that $[x]$ is dense in $\overline{[y]}$, i.e., $\overline{[y]}=\overline{[x]}$. Let $C=\overline{[y]}$. Since $[x]$ is a Baire space, $[x]$ is not meager in itself, and hence $[x]$ is not meager in $C$. Since $[x]$ is Borel, $[x]$ is almost open in $C$ so $[x] \cap U \cap C$ is comeager in $U \cap C$ for some $U$. Since $[y]$ is dense in $C,[y] \cap U \cap C \neq \varnothing$. Since $[y]$ is a Baire space, $[y] \cap U \cap C$ is not meager in $C$. But $[y] \cap U \cap C \cong U \cap C \sim[x]$ which is meager in $C$. This contradiction establishes the claim and, thereby, the lemma.

When $\mathscr{S} \cong A_{\tau}$ we say that $E$ is a $\gamma$-decomposition for $(\boldsymbol{Y}, \mathscr{H})$. We assume that this is the case, $\gamma \geqq 0$. Thus we are in a situation similar to subsection A.

We need an operator analogous to Vaught's 4 . Our solution is a kind of local version of the transform, cf. the remarks in [8] and [9] where such a version was described. For $B \subseteq Y, U \in \mathscr{H}$, we define

$$
B^{\Delta U}=\{y: U \cap B \cap[y] \text { is not meager in }[y]\}, \quad B^{\Delta}=B^{\Delta Y} .
$$

We have for $B, B_{i}$ Borel in $Y$ (and, by convention, $U, V \in \mathscr{H}$ )

$$
\begin{gathered}
B^{\Delta U} \cong(B \cap U)^{+} \\
V^{\Delta U}=(U \cap V)^{+} \\
\left(\bigcup_{i \in \omega} B_{i}\right)^{\Delta U}=\bigcup_{i \in \omega} B_{i}^{\Delta U} \\
(\sim B)^{\Delta U}=\bigcup_{V \in U}\left(V^{+} \sim B^{\Delta V}\right)
\end{gathered}
$$

(11) $B^{\Delta}$ is invariant and if $S$ is invariant then $(S \cap B)^{4}=S \cap B^{4}$.

The proofs of (7)-(11) are essentially similar to corresponding arguments in [11]. For completeness we indicate them here. 
For (7): The empty set is meager in any space so a nonmeager subset of $[y]$ is nonempty. Thus, for any $y, y \in B^{U} \Rightarrow U^{\Delta} \cap B \cap[y] \neq \varnothing$.

For (8): By (7) it suffices to check inclusion from right to left. Since $[y]$ is a Baire space, any nonempty open subset of $[y]$ is nonmeager. Thus, $U \cap B \cap[y] \neq \varnothing \Rightarrow y \in B^{\Delta U}$.

For (9): Using the fact that a countable union of meager sets is meager, note the equivalence $y \in\left(\mathbf{U}_{i} B_{i}\right)^{\Delta U} \Leftrightarrow U \cap \mathbf{U}_{i} B_{i} \cap[y]$ is nonmeager $\Leftrightarrow(\exists i)\left(U \cap B_{i} \cap[y]\right.$ is nonmeager $) \Leftrightarrow y \in \mathbf{U}_{i}\left(B_{i}{ }^{4 U}\right)$.

For (10): Since $B$ is a Borel set, $[y] \cap B$ is almost open in $[y]$ for each $y$; say $B \cap[y]$ is congruent to $C_{y}$ modulo a [y]-meager set, where $C_{y}$ is closed in $[y]$. Let $[y] \sim C_{y}=O_{y} \cap[y]$ where $O_{y}$ is open in $Y$. Then making repeated use of the fact that $[y]$ is a Baire space and $\mathscr{H}$ is a basis, we compute

$$
\begin{aligned}
y \in(\sim B)^{\Delta U} & \Longrightarrow(U \sim B) \cap[y] \text { is nonmeager in }[y] \\
& \Longleftrightarrow\left(U \cap O_{y}\right) \cap[y] \text { is nonmeager in }[y] \\
& \Longleftrightarrow\left(U \cap O_{y} \cap[y]\right) \text { is nonempty } \\
& \Longleftrightarrow(\exists V \subseteq U)(V \cap[y] \neq \varnothing \text { and } V \subseteq U)\left(V \cap[y] \neq \varnothing \text { and } V \cap C_{y}=\varnothing\right) \\
& \Longleftrightarrow(\exists V \leqq U)\left(V \cap[y] \neq \varnothing \text { and } V \cap C_{y} \text { is meager in }[y]\right) \\
& =(\exists V \subseteq U)(V \cap[y] \neq \varnothing \text { and } V \cap B \text { is meager in }[y]) \\
& =y \in \bigcup_{V \subseteq U}\left(V^{+} \sim\left(B^{\prime V}\right)\right) .
\end{aligned}
$$

For (11): The invariance of $B^{\Delta}$ (and of any $B^{\Delta U}$ ) is apparent from the definition. If $S$ is invariant, then for any $y$, either $[y] \cap$ $S=\varnothing$ or $[y] \subseteq S$. Thus,

$$
\begin{aligned}
y \in(S \cap B)^{\Delta} & \Longleftrightarrow S \cap B \cap[y] \text { is nonmeager in }[y] \\
& \Longleftrightarrow[y] \subseteq S \text { and } B \cap[y] \text { is nonmeager in }[y] \\
& \Longleftrightarrow y \in S \text { and } y \in B^{\Delta} .
\end{aligned}
$$

Corresponding to 3.1 we have the somewhat stronger:

Lemma 3.3. For all $\alpha$, if $B \in S_{\alpha}$ then $B^{\Delta U}$ is a countable union of invariant $A_{\gamma+\alpha}$ sets.

Proof. We proceed by induction on $\alpha$.

For $\alpha=0$ note that any open set $O$ can be written $O=\bigcup_{i<\omega} V_{i}$ with each $V_{i} \in \mathscr{H}$. Then by (9) and (8), $O^{\Delta U}=\left(\mathbf{U}_{i} V_{i}\right)^{\Delta U}=\mathbf{U}_{i}\left(V_{i}^{\Delta U}\right)=$ $\mathrm{U}_{i}\left(V_{i} \cap U\right)^{+}$. Since $\mathscr{H}$ is a basis, $V_{i} \cap U \in \mathscr{H}$. Since $E$ is a $\gamma$ decomposition for $(Y, \mathscr{H})$, each $\left(V_{i} \cap U\right)^{+} \in A_{\gamma}$. For $\alpha \geqq 1$, write $B=\bigcup_{i \in \omega} \sim B_{i}$ with each $B_{i} \in \bigcup_{B<\alpha} S_{\beta}$ and note that 


$$
B^{\Delta U}=\bigcup_{i}\left(\sim B_{i}\right)^{\Delta U}=\bigcup_{i} \bigcup_{\Xi U}\left(V^{+} \cap B_{i}^{\Delta V}\right) .
$$

As in the introduction, we write $\gamma^{*}=\sup \{\gamma+\beta: \beta<\gamma\}$.

THEOREM 3.5. Suppose $E$ is a $\gamma$-decomposition for $(\boldsymbol{Y}, \mathscr{H}), \gamma \geqq 0$. Then there is a $\gamma^{*}$-Borel selector for $E$ on $\boldsymbol{Y}$.

Proof. First consider the case $\gamma=0$. In this case Theorem 1.1 (or rather its classical antecedent) can be applied directly. By 3.3 each $[y]$ is an intersection of clopen sets and, hence, is closed. Set $X=Y / E, \mathscr{D}=\{\pi(C): C$ is an $E$-invariant clopen set $\}$, and for each open $O \cong Y$ set

$$
\begin{aligned}
O^{*} & =\pi\left(O^{+}\right)=\{[y]:[y] \cap O \neq \varnothing\} \\
& =\mathbf{U}\left\{\pi\left(U^{+}\right): U \in \mathscr{H} \text { and } U \subseteq O\right\} .
\end{aligned}
$$

Theorem 1.1 then applies to provide a choice function $f: Y / E \rightarrow Y$ which is $\mathscr{D}_{\sigma}$-measurable. Define a selector $s$ by setting $s=f \circ \pi$. Then for any open $O, s^{-1}(O)$ is of the form $\pi^{-1}\left(\mathbf{U}_{i} \pi\left(C_{i}\right)\right)=\bigcup_{i} \pi^{-1} \pi\left(C_{i}\right)$ where each $C_{i}$ is invariant clopen. Since $\pi^{-1} \pi(C)=C$ for any invariant set $C$, each $s^{-1}(O)$ is open and $s$ is continuous as required.

Now suppose $\gamma \geqq 1$. As before, we imitate the above argument by introducing a new topology and substituting $\Delta$ for + . By 2.3 we can choose a countable collection $\mathscr{C} \subseteq \bigcup_{\beta<r} A_{\beta}$ such that $\mathscr{S} \cup$ $\mathscr{C} \cup \mathscr{H}$ generates a Polish topology $\mathscr{T}^{\prime}$ on $Y$. For $O \cong Y^{\prime}=\left(Y, \mathscr{T}^{\prime}\right)$ we define $O^{\sharp}=\pi\left(O^{\Delta}\right)$ using our new $\Delta$ operator and, using (7) and (9), again observe that \# is a countably additive semisaturation operator for the identity map $Y / E \rightarrow \mathscr{P}\left(\boldsymbol{Y}^{\prime}\right)$.

Set $\mathscr{D}=\left\{\pi(A): A\right.$ is an invariant $A_{\alpha}$ set $\}$. We claim

If $O$ is open in $Y^{\prime}$, then $O^{\ddagger} \in \mathscr{D}_{\sigma}$.

Let $\mathscr{H}^{\prime}$ be the collection of sets of the form $B \cap S$ where $B$ is a finite intersection of elements of $\mathscr{C} \cup \mathscr{H}$ and $S$ is a finite intersection of elements of $\mathscr{S}$. Since $\mathscr{H}^{\prime}$ is a basis for $Y^{\prime}$ and $\#$ is countably additive, it suffices to check that (12) holds for $O \in \mathscr{H}^{\prime}$. Given $O=$ $B \cap S$ with $B, S$ as above we have $O^{\sharp}=\pi(B \cap S)^{4}=\pi\left(B^{\Delta} \cap S\right)$. Noting that $B \in S_{\beta}$ for some $\beta<\gamma$, we may apply 3.3 to conclude that $B^{4}$ is a countable union of invariant $A_{\gamma+\beta}$ sets. Since $S$ is invariant $A_{\gamma}, B^{\Delta} \cap S$ is a countable union of invariant $A_{\gamma+\beta}$ sets. Since $\gamma+$ $\beta \leqq \gamma^{*}$ and projection commutes with union, $\pi\left(B^{3} \cap S\right) \in \mathscr{D}_{\sigma}$ as required, establishing (12).

As in 3.2 we note that each $[y]$ is closed in $Y^{\prime}$. Thus, we are again in a position to apply 1.1 to obtain a $\mathscr{D}_{\sigma}$-measurable choice function $f: Y \mid E \rightarrow Y^{\prime}$. As before, the map $s=f \circ \pi$ is a selector. In this case, however, it follows directly from our definition of $\mathscr{D}$ that 
$s$ is an $\alpha$-Borel map from $Y$ to $Y^{\prime}, \alpha$ fortior $i$ an $\alpha$-Borel map from $Y$ to $Y$. The theorem is proved.

Corollary 3.6. Suppose $\boldsymbol{A}$ is an $A_{\gamma}$ subspace of $\boldsymbol{Y}$ with basis $\mathscr{H}^{\prime}=\{U \cap A: U \in \mathscr{K}\}$. Suppose $E^{\prime} \subseteq \boldsymbol{A} \times \boldsymbol{A}$ is a $\gamma$-decomposition for $\left(\boldsymbol{A}, \mathscr{Z}^{\prime}\right)$. Then there is an $\gamma^{*}$-Borel selector for $E^{\prime}$ on $\boldsymbol{A}$.

Proof. When $\gamma \leqq 1$, we can apply 3.5 directly to the Polish space $\boldsymbol{A}$. For $\gamma>1$, set $E=E^{\prime} \cup\{(y, y): y \in Y\}$. Then for any $U \in \mathscr{L}, U^{+E}=(U \sim A) \cup(U \cap A)^{+\prime^{\prime}} \in A_{\gamma}$. It follows easily that $E$ is a $\gamma$-decomposition for $(Y, \mathscr{C})$. 3.5 provides an $\gamma^{*}$-Borel selector for $E$ whose restriction to $A$ is an $\gamma^{*}$-Borel selector for $E^{\prime}$.

As an application of 3.7 we can derive a multifunction version. ${ }^{1}$

Corollary 3.7. Let $\gamma \geqq 1$ and let $\mathbb{Z}$ be Polish.

Suppose $G \leqq Z \times Y$ is an $A_{r}$ set such that

(i) For eash $z \in \mathbb{Z}, G_{z}=\{y:(z, y) \in G\}$ is $G_{\hat{o}}$

(ii) For every $U \in \mathscr{K},\left\{z: G_{z} \cap U \neq \varnothing\right\}$ is $A_{\gamma}$. Then there is an $M_{\gamma^{*}}$ set which uniformizes $G$.

Proof. Let $\mathscr{K}^{\prime \prime}=\{O \times U: O$ is open in $Z$ and $U \in \mathscr{K}\}, \mathscr{K}^{\prime \prime}=$ $\left\{B \cap G: B \in \mathscr{K}^{\prime \prime}\right\}$. Set $E=\left\{(z, y),\left(z^{\prime}, y^{\prime}\right) \in G \times G: Z=Z^{\prime}\right\}$. For $W=$ $(O \times U) \cap G \in \mathscr{H}^{\prime}$ we have $W^{+E}=\left\{(z, y) \in G: z \in O\right.$ and $\left.G_{y} \cap U \neq \varnothing\right\} \in$ $A_{\gamma}$ so $E$ is a $\gamma$-decomposition for $\left(G, \mathscr{X}^{\prime}\right)$. Let $s$ be the $\gamma^{*}$-Borel selector for $E$ on $G$ provided by 3.6. Then $T_{s}=\{(z, y) \in G:(z, y)=$ $s((\approx, y))\}$ is an $M_{\gamma *}$ uniformization for $G$.

\section{REFERENCES}

1. J. P. Burgess, A selection theorem for group actions, Pacific J. Math., 80 (1979), 333-336.

2. E. Effros, Transformation groups and C-algebras, Ann. Math., 81 (1965), 39-55.

3. R. Kallman and R. D. Mauldin, A cross-section theorem and an application to $C^{*}$. algebras, AMS Proceedings, 61 (1978), 57-61.

4. K. Kuratowski, Topology, vol. 1, Academic Press, New York, 1966.

5. K. Kuratowski and A. Maitra, Some theorems on selectors and their applications to semicontinuous decompositions, Bull. Acad. Polon. Sci. Ser. Sci. Math. Astron. Phys., 22 (1974), 887-891.

6. K. Kuratowski and C. Ryll-Nardzewski, A general theorem on selectors, Bul. Acad. Polon. Sci. Ser. Sci. Math. Astron. Phys., 13 (1965), 397-403.

7. D. E. Miller, On the measurability of orbits in Borel actions, AMS Proc., 63 (1977), 165-170.

8. — A selector for equivalence relations with $G_{\tilde{o}}$ orbits, AMS Proc., to appear.

9. _— Review of V. Harnik, "Approximation theorems and model theoretic forcing” in Math. Reviews, 55 (1978).

1 The author is grateful to John Burgess for this remark. 
10. S. M. Srivastava, Selection theorems for $G_{\delta}$-valued multifunctions, Trans. Amer. Math. Soc., 254 (1979), 283-293.

11. R. L. Vaught, Invariant sets in topology and logic, Fund. Math., 82 (1974/5), 269-294.

Received November 7, 1978 and in revised form April 22, 1980. Research supported by NSF Grant 78-02845.

University of Illinors-Chicago Circle

ChicAgo, IL 60680 


\section{PACIFIC JOURNAL OF MATHEMATICS}

\section{EDITORS}

DoNALD BABBITT (Managing Editor)

University of Galifornia

Los Angeles, California 90024

HUgo RossI

University of Utah

Salt Lake City, UT 84112

C. C. MOORE AND ANDREW OGG

University of California

Berkeley, CA 94720
J. DugunduI

Department of Mathematics

University of Southern California

Los Angeles, California 90007

R. Finn and J. Milgram

Stanford University

Stanford, California 94305

\section{ASSOCIATE EDITORS}
E. F. BeCKenbaCh
B. H. NeumanN
F. WOLF
K. YoSHIDA

\section{SUPPORTING INSTITUTIONS}

UNIVERSITY OF BRITISH COLUMBIA

CALIFORNIA INSTITUTE OF TECHNOLOGY

UNIVERSITY OF CALIFORNIA

MONTANA STATE UNIVERSITY

UNIVERSITY OF NEVADA, RENO

NEW MEXICO STATE UNIVERSITY

OREGON STATE UNIVERSITY

UNIVERSITY OF OREGON
UNIVERSITY OF SOUTHERN CALIFONIA STANFORD UNIVERSITY UNIVERSITY OF HAWAII UNIVERSITY OF TOKYO UNIVERSITY OF UTAH WASHINGTON STATE UNIVERSITY UNIVERSITY OF WASHINGTON 


\section{Pacific Journal of Mathematics}

\section{Vol. 91, No. $1 \quad$ November, 1980}

Harvey Leslie Abbott, Extremal problems on nonaveraging and nondividing

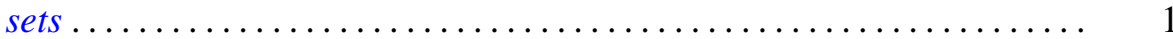

Marine Bruce Abrahamse and Stephen D. Fisher, Mapping intervals to

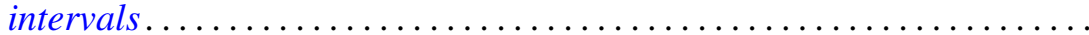

William Wells Adams, The best two-dimensional Diophantine

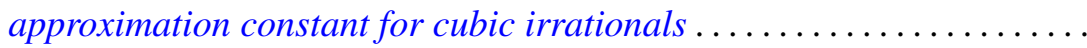

Marilyn Breen, A quantitative version of Krasnosel'skiu 's theorem in

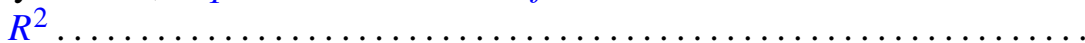

Stephen LaVern Campbell, Linear operators for which $T^{*} T$ and $T T^{*}$

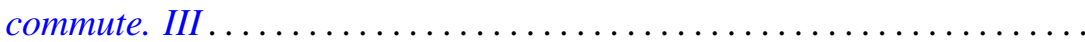

Zvonko Cerin, On cellular decompositions of Hilbert cube manifolds ......

J. R. Choike, Ignacy I. Kotlarski and V. M. Smith, On a characterization

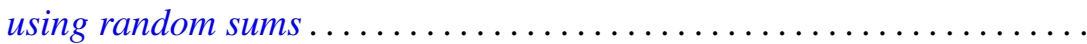

Karl-Theodor Eisele, Direct factorizations of measures .............. 79

Douglas Harris, Every space is a path component space ............. 95

John P. Holmes and Arthur Argyle Sagle, Analytic H-spaces, Campbell-Hausdorff formula, and alternative algebras.............

Richard Howard Hudson and Kenneth S. Williams, Some new residuacity criteria ..........................................

V. Karunakaran and Michael Robert Ziegler, The radius of starlikeness for a class of regular functions defined by an integral ....

Ka-Sing Lau, On the Banach spaces of functions with bounded upper

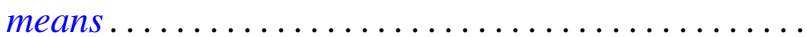

Daniel Paul Maki, On determining regular behavior from the recurrence formula for orthogonal polynomials................

Stephen Joseph McAdam, Asymptotic prime divisors and going down...

Douglas Edward Miller, Borel selectors for separated quotients ..

Kent Morrison, The scheme of finite-dimensional representations of an algebra

Donald P. Story, A characterization of the local Radon-Nikodým property by tensor products

Arne Stray, Two applications of the Schur-Nevanlinna algorithm ...

N. B. Tinberg, The Levi decomposition of a split $(B, N)$-pair ...

Charles Irvin Vinsonhaler and William Jennings Wickless, A theorem on quasi-pure-projective torsion free abelian groups of finite rank... 